\title{
Endovascular Treatment of Deep Hemorrhagic Brain Arteriovenous Malformations with Transvenous Onyx Embolization
}

\author{
A. Consoli, L. Renieri, S. Nappini, N. Limbucci, and S. Mangiafico
}

\begin{abstract}
SUMMARY: Brain AVMs are a rare cause of cerebral hemorrhage and SAH, and their treatment is still debated. The aim of this study was to describe a novel endovascular approach with transvenous embolization of deep hemorrhagic brain AVMs. Five patients ( 3 females, 2 males; mean age, 33.2 years) underwent a transvenous embolization of a deep hemorrhagic brain AVM at our institution between February and April 2012. Clinical and angiographic data at the end of the procedure and after 6 months are reported. All the AVMs were completely obliterated, and no intra- or postprocedural complications occurred. The clinical outcome was unchanged or improved in all patients. Transvenous endovascular treatment may be considered in small, hemorrhagic, and deep AVMs with single deep drainage in those cases in which neurosurgical and radiosurgical treatment might not be indicated.
\end{abstract}

ABBREVIATIONS: $\mathrm{ACA}=$ anterior cerebral artery; $\mathrm{AchoA}=$ anterior choroidal artery; $\mathrm{bAVMs}=$ brain $\mathrm{AVM} ; \mathrm{mRS}=$ modified Rankin Scale; $\mathrm{PCA}=$ posterior cerebral artery

B rain AVMs are a rare cause of cerebral hemorrhage or SAH, and the therapeutic strategy is still debated. Several studies reported in the literature compared the results of endovascular, neurosurgical, and radiosurgical series, considering both single or associated treatments. ${ }^{1-4}$ Concerning the embolization of bAVMs, the conventional transarterial treatment showed encouraging results in terms of safety and effectiveness, even if the results are less satisfactory than the surgical ones. ${ }^{5-8}$ However, in those cases in which the surgical approach may expose the patient to high risk, such as in deep localizations or eloquent areas, the endovascular and/or radiosurgical treatment may provide better clinical results. ${ }^{9}$ Furthermore, the slow effects of radiosurgery may be considered a limitation in hemorrhagic bAVMs because the risk of an early rebleeding would not be reduced. The evolution of endovascular treatment with the introduction of embolizing agents that progressively occlude the lumen of arteries and veins (Onyx 18; ev3-Covidien, Irvine, California) and detachabletip microcatheters has allowed interventional neuroradiologists

Received October 24, 2012; accepted after revision November 26.

From the Interventional Neuroradiology Unit, Careggi University Hospital, Florence, Italy.

Drs Arturo Consoli and Salvatore Mangiafico were responsible for the study concepts, the manuscript preparation, and the final review. Drs Leonardo Renieri, Sergio Nappini, and Nicola Limbucci contributed to the data acquisition and the manuscript review.

Please address correspondence to Arturo Consoli, MD, Interventional Neuroradiology Unit, Careggi University Hospital, Largo Brambilla 3, 50134, Firenze, Italy;

e-mail: onemed21@gmail.com

http://dx.doi.org/10.3174/ajnr.A3497 to plan different strategies to obtain safe curative embolizations of bAVMs. The aim of this work was to describe a preliminary experience with transvenous embolization in a small series of patients with deep hemorrhagic bAVMs.

\section{MATERIALS AND METHODS}

Five patients ( 3 females, 2 males; mean age, 33.2 years) with deep hemorrhagic bAVMs underwent endovascular treatment with Onyx 18 by using a transvenous approach at our institution between February and April 2012. Patient characteristics are summarized in the Table. All patients were admitted or referred to our Interventional Neuroradiology Unit after a CT scan showed an SAH or parenchymal hematoma in atypical locations and a DSA examination documented the presence of a bAVM with deep venous drainage (the size of the nidus was evaluated in centimeters: height $\times$ length $\times$ width). In 4 patients, the AVMs had never been treated, and in 1 patient, the residual nidus of a previously subtotally occluded AVM was responsible for a late bleeding 3 years later.

Our interventional neuroradiology and neurosurgical teams discussed each case, and the endovascular treatment was preferred. Endovascular treatment was chosen after considering the risks of the surgical approach and the transvenous approach because of the presence of arterial feeders made up of short perforator branches arising from functional arteries (AChoA and the P2 segment of PCA in 3 cases, the posterolateral choroidal artery in 1 case, and the anteroinferior cerebellar artery in 1 case). The treatment was scheduled at least 3 weeks after the hemorrhage (average latency was 61.8 days). All the endovascular procedures were performed with the patient under general anesthesia, without systemic heparinization (heparin was ad- 
Patient characteristics

\begin{tabular}{|c|c|c|c|c|c|c|c|c|}
\hline $\begin{array}{l}\text { Patient } \\
\text { No. }\end{array}$ & $\begin{array}{l}\text { Age } \\
\text { (yr), } \\
\text { Sex }\end{array}$ & $\begin{array}{c}\text { First } \\
\text { Treatment/ } \\
\text { Residual }\end{array}$ & Localization, Side & $\begin{array}{l}\text { Venous } \\
\text { Drainage }\end{array}$ & $\begin{array}{l}\text { Vascular } \\
\text { Approach }\end{array}$ & $\begin{array}{l}\text { Nidus } \\
\text { Size } \\
\text { (cm) }\end{array}$ & $\begin{array}{l}\text { Angiographic } \\
\text { Result }\end{array}$ & $\begin{array}{l}\text { Intra- or Post } \\
\text { procedural } \\
\text { Complications }\end{array}$ \\
\hline 1 & $36, \mathrm{~F}$ & First treatment & Paraventricular, left & Deep, single & Transvenous & $<3$ & Complete occlusion & None \\
\hline 2 & $23, \mathrm{~F}$ & First treatment & $\begin{array}{l}\text { Thalamomesencephalic, } \\
\text { left }\end{array}$ & Deep, single & Transvenous & $<3$ & Complete occlusion & None \\
\hline 3 & $11 \mathrm{~F}$ & First treatment & $\begin{array}{l}\text { Pontocerebellar angle, } \\
\text { left }\end{array}$ & Deep, single & Combined & $<3$ & Complete occlusion & None \\
\hline 4 & $36, M$ & First treatment & Paraventricular, left & Deep, single & Combined & $<3$ & Complete occlusion & None \\
\hline 5 & $61, \mathrm{M}$ & Residual & $\begin{array}{l}\text { Medial temporopolar, } \\
\text { left }\end{array}$ & $\begin{array}{l}\text { Deep (single) and } \\
\text { superficial (single) }\end{array}$ & Combined & $<3$ & Complete occlusion & None \\
\hline
\end{tabular}

ministered only in the perfusion sac). All arterial and venous accesses were performed from the femoral artery and vein. A triaxial system was used in the transvenous approach: a 6F guide catheter (Envoy; Codman Neurovascular, Raynham, Massachusetts), an intermediate catheter (DAC 0.044; Concentric Medical, Mountain View, California), and a 3-cm detachable-tip microcatheter (Apollo; ev3-Covidien). A second microcatheter (Apollo) was positioned in all cases in the direct main arterial feeder to the AVM to obtain a selective study of the nidus and its venous drainage without the overlap with other vascular structures and to perform intraprocedural controls during the transvenous embolization.

In 3 cases, a combined embolization (tranvenous + transarterial) was performed, and in the other 2 cases, Onyx was injected only from the venous side. The "plug and push" technique was used to inject Onyx retrogradely from the origin of the intranidal portion of the draining vein into the nidus and subsequently into the arterial feeders by using a roadmap view. The fractionated injection of Onyx (average volume injected, $2.5 \mathrm{~mL}$ ) was performed in 60-90 minutes. The procedure was considered completed when arteriovenous shunts were no longer recognizable during the angiographic controls, the draining vein was completely occluded, and a reflux within the arterial feeders direct to the nidus was obtained. The final phases of the embolizations, when the proximal segment of the draining vein was occluded, were performed under controlled hypotension. All patients underwent CT immediately after the procedure, and a delayed awakening was performed in the Neurosurgical Intensive Care Unit approximately 6-12 hours later. A clinical neurologic examination was performed in all cases at discharge; at 6 months, angiographic follow-up was performed in $4 / 5$ patients. IV dexamethasone, $8 \mathrm{mg} /$ day, was administered for 1 week after the procedure to prevent possible postoperative focal brain edema and perilesional swelling.

\section{RESULTS}

In all 5 cases, complete occlusion of the AVM was obtained without intra- or postprocedural complications and the CT performed at the end of the procedures showed no hemorrhage. The clinical status of all patients was unchanged or improved at discharge and after 6 months. A brief description of each case is reported below.

\section{Case 1}

A 36-year-old woman was admitted to our institution after the onset of acute headache, aphasia, and severe hemiparesis. A CT scan showed a $5 \times 4 \mathrm{~cm}$, deep, left temporoparietal hematoma with perilesional edema. The patient immediately underwent a DSA evaluation, which documented a small $(2.5 \times 2 \times 1.5 \mathrm{~cm})$, deep, retroinsular, thalamic-pulvinar, periventricular, leftsided bAVM. The arterial feeders originated from the distal segment of the AchoA, from the MCA (insular perforating arteries), and from the ACA (artery of the splenium of the corpus callosum); the AVM was drained through the deep venous system (single drainage: superior choroidal vein toward the internal cerebral vein). The case was discussed with our neurosurgical team, and we decided to proceed with the evacuation of the hematoma, which was considered life-threatening, and the removal of the AVM. After the operation, DSA evaluation showed that the superficial part of the AVM, fed from branches originating from the MCA and the ACA, was removed and a deep residual portion of the AVM was still present (Fig $1 A,-B$ ). The endovascular treatment was scheduled when the patient was clinically stable. A microcatheter was positioned through the straight sinus and the internal cerebral vein within the intranidal segment of the superior choroidal vein. A second microcatheter was positioned within the AchoA to perform a superselective study of the AVM and further controls during the procedure (Fig $1 C,-D$ ). The AVM was completely obliterated, and the transvenous injection of 1.5 $\mathrm{mL}$ of Onyx (Fig $1 E,-F$ ) was necessary to obtain the retrograde filling of the arterial feeders at the end of the procedure. The final angiograms, the postprocedural CT scan, the MRI-MRA (1 week later), and the 6-month DSA follow-up confirmed the complete obliteration of the AVM without complications (Fig 2). The patient was discharged with a slight dysarthria (mRS score, 1) and, after 6 months, was asymptomatic (mRS score, 0$)$.

\section{Case 2}

A 23-year-old female patient was referred to our institution after a previous hemorrhage ( 1 year before) from a small $(2 \times 1.5 \times 1.5$ $\mathrm{cm}$ ), left, subthalamomesencephalic AVM occurred a few days after the positioning of ventriculo-peritoneal drainage in a thirdventricular cyst. Several radiosurgical opinions were sought with negative responses. The neurologic examination at admission documented a moderate right hemiparesis, a slight left seventh cranial nerve palsy, left ptosis, and a partial deficit of the homolateral cranial nerve III with left mydriasis. Distal branches of the AchoA and small thalamogeniculate perforators from the P2 segment of the posterior cerebral artery fed the AVM with a single, deep venous drainage through the left peduncular vein, a tribu- 

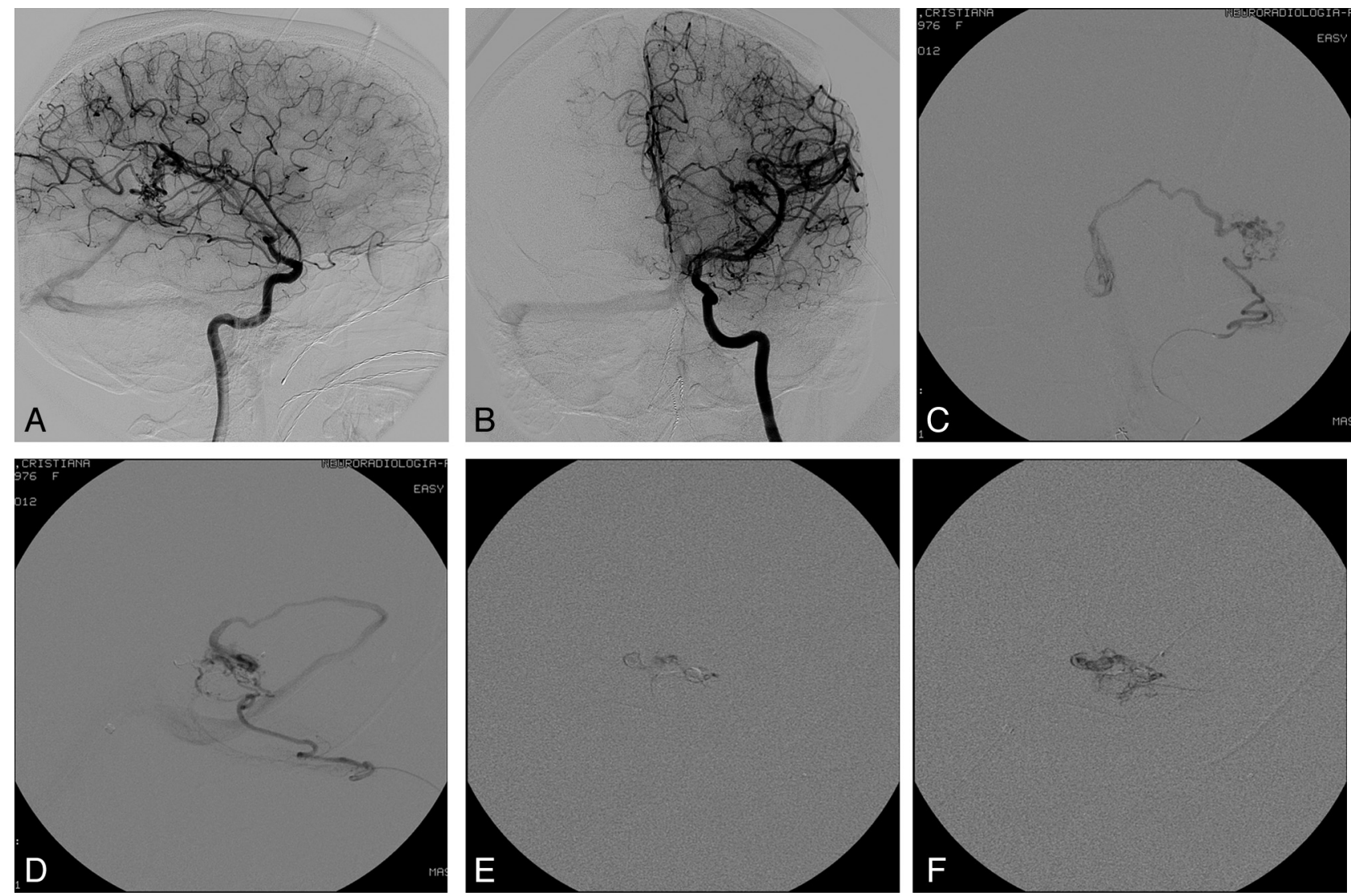

FIG 1. Case 1. Pretreatment DSA examination in anteroposterior (AP; $A$ ) and lateral (B) views. Visualization of the angioarchitecture of the AVM through a superselective injection within the AchoA in AP $(C)$ and lateral $(D)$ projections. Intranidal progression of Onyx during transvenous injection from the intranidal portion of the main draining vein $(E$ and $F)$.

tary of the basal vein of Rosenthal. A bifemoral arterial access was used. A microcatheter was placed within the AchoA, at the level of the plexal point and immediately after the origin of perforators, directed toward the internal capsule (visualized with injections within the AchoA), to have direct control of the nidus. A balloon (HyperGlide $4 \times 15$; ev3-Covidien) was positioned within the PCA and inflated each time Onyx penetrated the nidus to avoid retrograde filling of the artery through the perforators. A venous femoral access was used to reach the intranidal segment of the draining vein. The AVM, the draining vein, and the arterial feeders were occluded, preserving the PCA and the AchoA, with the injection of $1.8 \mathrm{~mL}$ of Onyx. A postprocedural CT scan showed no new ischemic or hemorrhagic lesions, and MR imaging performed 1 week later was negative for recent cerebral thalamopeduncular ischemic lesions. A 6-month DSA follow-up showed stable occlusion of the nidus. The patient was discharged with unchanged neurologic examination findings (mRS score, 3 ), with a slight motor improvement after 6 months.

\section{Case 3}

An 11-year-old girl was referred to our interventional neuroradiology unit after a hemorrhage in the posterior cranial fossa secondary to a small AVM $(1 \times 1 \times 1.5 \mathrm{~cm})$ of the left pontocerebellar angle fed by the rostrolateral branch of the anteroinferior cerebellar artery and drained through a cortical ectatic cerebellar vein toward the superior petrous sinus. The case was discussed with our pediatric neurosurgical team, and the en- dovascular approach was chosen. At admission, the patient was asymptomatic. A combined treatment was performed: The nidus was subtotally obliterated with $1 \mathrm{~mL}$ of Onyx from the venous side; and the injection of $0.3 \mathrm{~mL}$ of Glubran (GEM, Viareggio, Italy) at $20 \%$ dilution from the arterial feeder completed the embolization. The AVM was also completely occluded after 6 months, and the patient was asymptomatic at discharge and during the clinical follow-up (mRS score, 0 ).

\section{Case 4}

A 36-year-old male patient was referred to our center after a CT scan and DSA detected a small $(1.5 \times 2 \times 2.5 \mathrm{~cm})$, hemorrhagic, left retrothalamic parasplenic AVM that caused a severe $\mathrm{SAH}$ with ventricular inundation. The patient was comatose and an external ventricular drainage was immediately positioned. On awakening, he was in a vegetative status, which slightly improved during the 7 following months (mRS score, 5). The multiple arterial feeders originated from the left posterior choroid plexus, and the single venous drainage was observed through a homolateral subependymal parachoroidal vein tributary of the atrial vein toward the basal vein of Rosenthal. The left posterolateral choroidal artery and the draining vein were catheterized; and combined alternate injections of Onyx ( $1.4 \mathrm{~mL}$ from the venous side and $0.8 \mathrm{~mL}$ from the posterolateral choroidal artery) were performed, obtaining the complete exclusion of the AVM (Figs 3 and 4). A postprocedural CT scan was negative for new ischemic or hemorrhagic lesions. In this patient, it was not possible to perform DSA control 

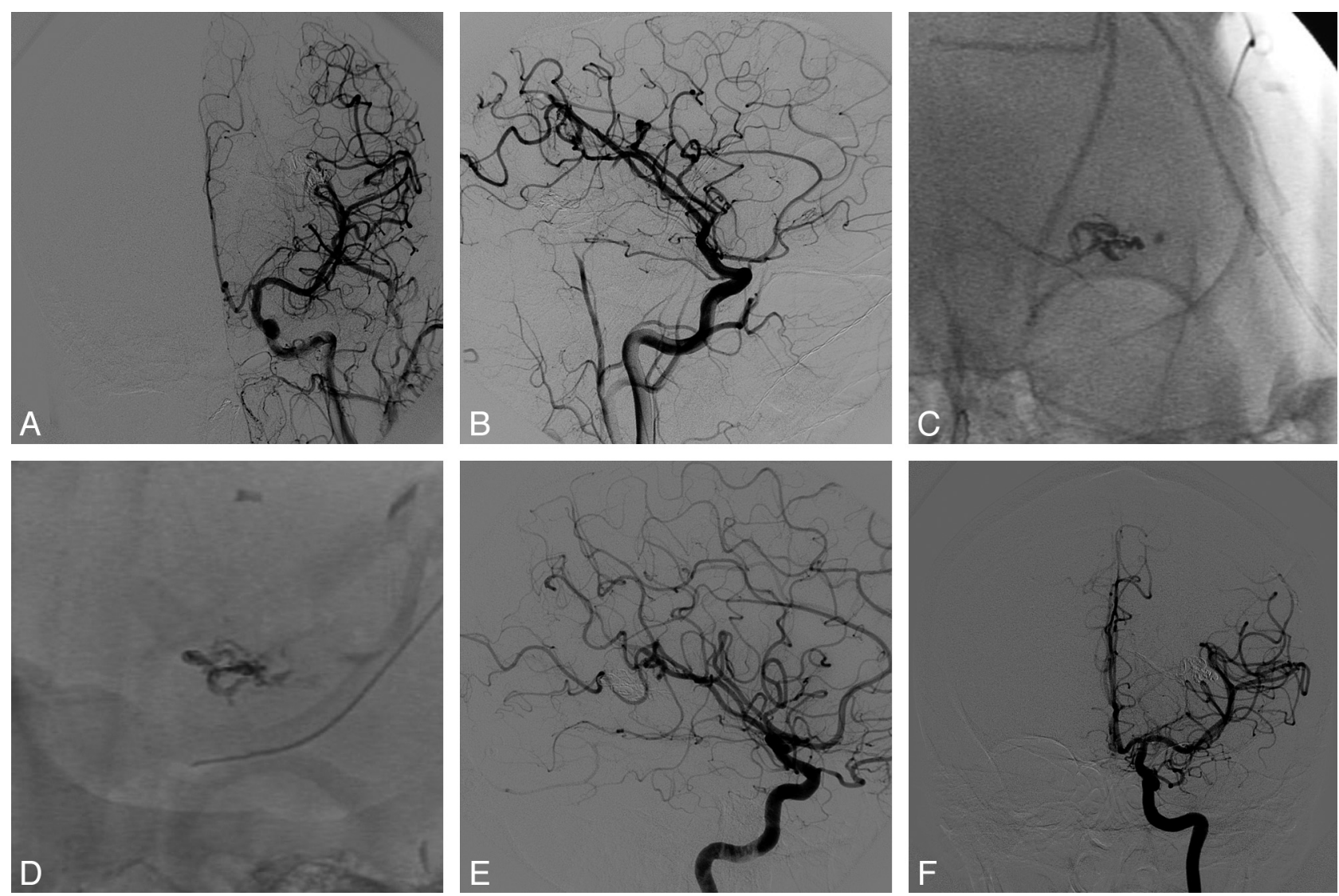

FIG 2. Case 1. Final angiograms at the end of the procedure in anteroposterior $(A P ; A)$ and lateral $(B)$ views. Onyx cast at the end of the procedure (DSA unsubtracted images) in AP $(C)$ and lateral $(D)$ views. Six-month DSA follow-up in AP $(E)$ and lateral $(F)$ projections confirming the complete and persistent occlusion of the AVM.

because his clinical condition was unchanged at discharge and after the 6-month clinical follow-up (mRS score, 5).

\section{Case 5}

A 61-year-old man with type 1 neurofibromatosis and Crohn disease was referred to our institution in 2009 after a brain MR imaging and MR angiography revealed the presence of a left, medium $(2 \times 3 \times 2.5 \mathrm{~cm})$, medial temporobasal AVM fed by the AchoA, middle meningeal artery, and MCA (temporal branches), drained through a temporobasal cortical vein and the anterior insular vein, a tributary of the first segment of the basal vein of Rosenthal. The AVM was treated with transarterial embolization, obtaining the subtotal exclusion of the nidus with a small remnant portion $(1.5 \times 1 \times 1 \mathrm{~cm})$, and radiosurgical completion was recommended. The patient did not undergo radiosurgery for personal reasons and because of the surgical removal of a mediastinal neurofibroma. In March 2012, he was admitted to our center because of a left temporal hemorrhage, which was detected with CT. At admission, the patient was in a stupor, aphasic, and severely hemiparetic (mRS score, 4). DSA evaluation was immediately performed and documented an increased volume of the remnant portion of the AVM $(3 \times 2 \times 2 \mathrm{~cm})$ with arterial feeders arising from the cisternal segment and from the plexal point of the left AchoA, from small perforators of the MCA (anterior temporal artery), from the PCA (anterior temporal branches), and minimally from the accessory meningeal artery. The plexiform nidus was drained predominantly through the deep venous system (basal vein of Rosenthal), and a small superficial drainage was observed through a temporal cortical vein tributary of the vein of Labbé. Endovascular treatment was preferred, and a combined approach was chosen. The accessory meningeal artery and the basal vein of Rosenthal were catheterized; after the occlusion of the minimal meningeal feeders, the nidus and the draining vein were completely obliterated with a transvenous intranidal injection of $6 \mathrm{~mL}$ of Onyx and retrograde filling of all the arterial feeders. The patient was discharged without neurologic deficits (mRS score, 0 ) and was asymptomatic even after 6 months. The DSA follow-up confirmed the complete obliteration of the AVM.

\section{DISCUSSION}

The results obtained in this small series raised some questions about critical issues that still remain debated, because to our knowledge, no series of patients with deep bAVMs treated with transvenous embolization has been reported in literature, though Massoud and Hademenos ${ }^{10}$ had previously proposed a very similar concept. The only case previously reported by Nguyen et $\mathrm{al}^{11}$ described a transvenous embolization of a hemorrhagic bAVM supplied by an en-passant arterial feeder. In this case, an intranidal pseudoaneurysm was angiographically detected and occluded with transvenous injections of Onyx 34 and Onyx 18, resulting in bAVM cure. The transvenous approach was chosen 

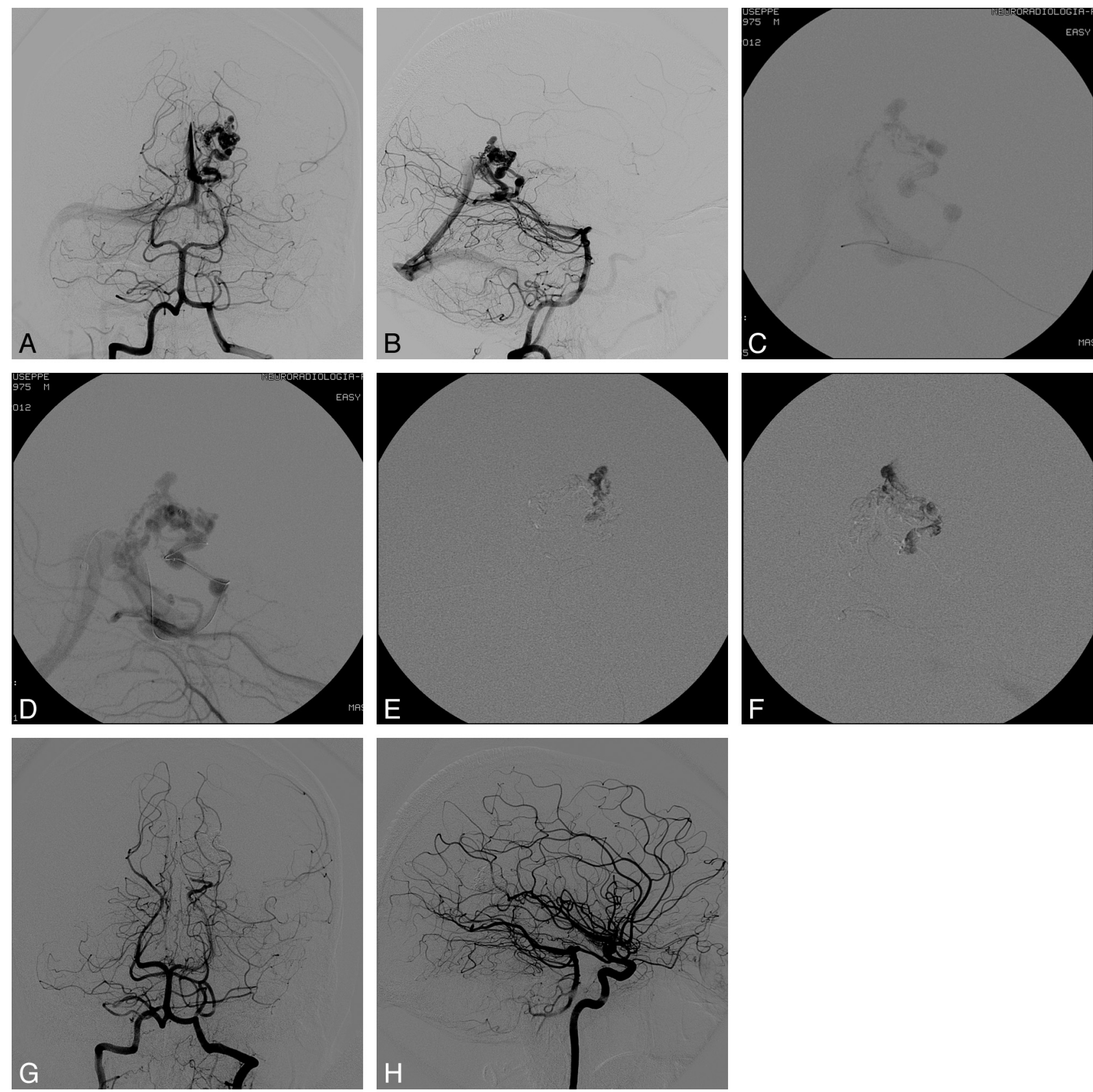

FIG 3. Case 4. Pretreatment DSA evaluation in anteroposterior (AP; $A$ ) and lateral (B) views. Visualization of the nidus and the draining venous system with transvenous injection through the microcatheter positioned at the origin of the draining vein $(C)$ and the intermediate catheter positioned within the straight sinus $(D)$. Intranidal penetration of Onyx through transvenous injection from the main venous drainage $(E$ and $F)$. DSA evaluation at the end of the procedure in $A P(G)$ and lateral $(H)$ views: The AVM is completely obliterated.

after an unsuccessful attempt of catheterization of the arterial feeder complicated by a branch occlusion secondary to a spasm. In our series, all the AVMs we treated with this approach were small $(<3 \mathrm{~cm})$, hemorrhagic, localized in deep territories with high risk for the surgical approach ( 1 in the pontocerebellar angle, 1 in the subthalamus and mesencephalon, 2 paraventricular thalamic-pulvinar AVMs, and 1 in the choroid fissure) and in eloquent areas. Furthermore, 4/5 AVMs had a single, deep drainage (only in case 5 was a superficial drainage present). Therefore, we considered these cases to be good targets for the transvenous approach.

The timing of treatment after the hemorrhage remains contro- versial. Although no evidence is reported in literature, to our knowledge, the presence of an intranidal pseudoaneurysm may be a reasonable indication for immediate endovascular treatment (target embolization). In our series, the aim of the transvenous approach was curative. A partial transvenous embolization would result in the occlusion of the venous outlet and in a potentially high risk of rebleeding from the residual nidus. The reason to wait for at least 3 weeks was to obtain the spontaneous reabsorption of the hematoma, which could compress and hide some portions of the nidus, and to encourage the healing of the bleeding point. Furthermore, no intra- or paranidal aneurysms were observed in our series after the DSA evaluation performed in the acute phase 


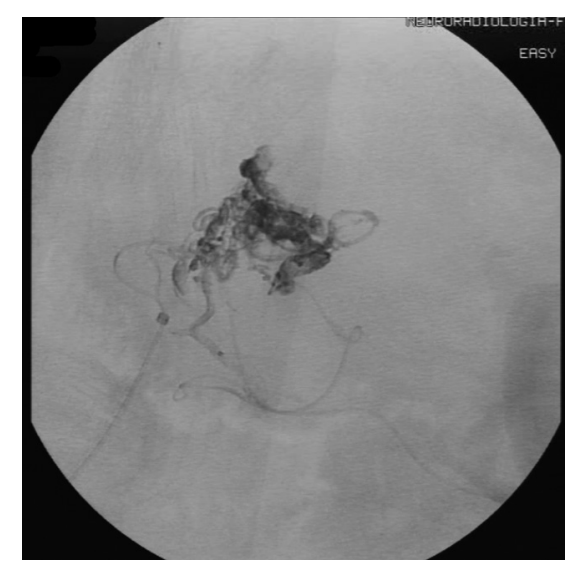

FIG 4. Case 4. Onyx cast at the end of the procedure (DSA unsubtracted image, lateral view).

of hemorrhage. The venous access was performed from the femoral vein and intracranially through the straight sinus in $4 / 5$ cases; the nidus was reached through basal vein of Rosenthal in 3 cases (cases 2, 4, and 5) and in 1 case from the superior choroidal vein (case 1). In the other case, the superior petrous sinus was used for the venous access and the nidus was reached through the vein of the great horizontal fissure of the cerebellum (case 3).

No technical difficulties were observed and no access-related complications occurred. In all the cases, the injection of Onyx was performed from the intranidal segment of the draining vein, adjacent to the nidus. We always decided to catheterize both the draining vein and the main arterial feeder for 2 reasons: to have a pure view of the nidus and, when possible, to perform superselective intraprocedural controls without overlapping vascular structures, though complete angiographic series were also obtained when injecting from the guiding catheter. A simultaneous injection in the carotid and vertebral territories from the guiding catheters was performed in 1 case, because the arterial feeders originated from the both the anterior and posterior circulation (case 2). In $3 / 5$ cases, transvenous embolization was associated with transarterial injection of Onyx to obliterate the peripheral parts of the nidus (cases 3, 4, and 5). However, when combined injections were performed, the nidus was predominantly occluded from the venous side. The decision to perform intra-arterial injections was case-dependent: In case of nonfunctional arteries (eg, the accessory meningeal artery or the distal posterolateral choroidal artery), the combined embolization with Onyx 18 (transarterial and transvenous, cases 4 and 5) was performed alternately to occlude the most peripheral portions of the nidus during the early phases of the procedure. In 1 case (case 3 ), the transarterial embolization was performed with $0.3 \mathrm{~mL}$ of Glubran exclusively during the final stages to achieve a rapid occlusion of residual peripheral portions of the bAVM. The transvenous injection of Onyx was used with the rationale of determining the retrograde occlusion of the nidus starting from the intranidal portion of the draining vein, where the dysplastic plexal venous shunts converge.

The transvenous embolization may be considered "centrifugal" because it determines the progression of Onyx from the central portions of the nidus toward the peripheral ones. The Onyx penetration through the intranidal dysplastic venous shunts, which are interposed between the draining vein and the periph- eral arterial feeders, occurs extensively when a stable plug is obtained within the origin of the draining vein. Onyx spreads toward the peripheral portions of the nidus until complete obliteration of the whole bAVM and its arterial feeders is achieved. During all the phases of the transvenous embolization, it is mandatory to have good control of the reflux along the draining vein. Onyx initially laminates along the venous wall without causing an immediate occlusion of the vessel. In this way, we were able to obtain a progressive reduction of the venous caliber and of the blood outflow, to determine a slow alteration of the hemodynamic balance during all the phases of the procedure.

We also persisted in injecting Onyx in roadmap view when the arteriovenous shunt was completely occluded during the angiographic controls, to obliterate all the small perinidal vessels that would not be recognizable at DSA evaluation. The venous reflux was $1-3 \mathrm{~cm}$. In all cases, it did not exceed the distal marker of the microcatheter and no difficulties were observed at retrieval. We were careful not to occlude the confluent veins into the main draining vein to avoid venous infarctions or bleeding secondary to hemodynamic alterations in the functional cerebral veins close to the bAVM. In all cases, the occlusion of a $1-$ to $3-\mathrm{cm}$ segment of the draining vein did not result in venous hemodynamic alterations, so we considered the deep occluded vein as functionally excluded from the cerebral circulation. That was confirmed in those patients who underwent MR imaging during the postprocedural hospitalization, because no perilesional edema was recognizable.

We considered the following as key points of this technique: the progressive and controlled lamination of Onyx within the draining vein; the transvenous rapid nidal occlusion and the retrograde filling of all the arterial feeders, which may prevent hemorrhagic complications. These complications may be secondary to hemodynamic imbalance due either to the restriction of the venous outlet caused by an early and uncontrolled obliteration of the distal part of the draining vein in the presence of a subtotal occlusion of the nidus, or because of the residual filling of portions of the nidus still fed by small feeders when the draining vein is already occluded. For this reason, we believe that the transvenous embolization may provide good control of the nidal occlusion and of the retrograde filling of all the arterial feeders. From this point of view, the mechanism of obliteration of a brain AVM may be similar to that occurring in dural arteriovenous fistulas: When the vein is occluded, retrograde occlusion of the arterial feeders is observed. Only in the presence of multiple, short, "comb" perforators supplying the AVM (case 2) was a balloon positioned within the PCA at the P2-P3 segment and inflated during the injections of Onyx to prevent reflux into the parent vessel. In the other cases, the perforators feeding the AVM were single and longer, and we had sufficient control of their retrograde filling.

The transvenous approach might not be indicated in medium and large AVMs with multiple venous drainage. Indeed, in these cases, the penetration of Onyx within the nidus would be slower with a venous outlet restriction when the main draining vein is occluded, with higher risk of venous hypertension within the accessory venous drainage and consequent possible venous bleeding. 
The main limitation of this series is the small number of patients, though we consider this study to represent only a preliminary experience. The possible indications, the operators' experience, and the selection of the patients represent some of many critical issues. However, further studies with larger populations are necessary to demonstrate the safety of this approach.

\section{CONCLUSIONS}

The transvenous embolization of deep bAVMs should still be described as an "experimental" treatment in small, hemorrhagic AVMs, when the surgical approach may be considered high risk and radiosurgery is not indicated. The feasibility of the technique and the possibility to achieve complete occlusion of the nidus with a single procedure, in those cases in which a conventional endovascular treatment is also challenging or not safe, may suggest that the transvenous approach is a further alternative therapeutic strategy in these types of bAVMs.

Disclosures: Salvatore Mangiafico-UNRELATED: Consultancy: ev3* and Codman Neurovascular. * Money paid to the institution.

\section{REFERENCES}

1. Fleetwood IG, Steinberg GK. Arteriovenous malformations. Lancet 2002;359:863-73

2. Mattle HP, Schroth G, Seiler RW. Dilemmas in the management of patients with arteriovenous malformations. J Neurol 2000;247:917-28

3. Richling B, Killer M, Al-Schameri AR, et al. Therapy of brain arte- riovenous malformations: multimodality treatment from a balanced standpoint. Neurosurgery 2006;59:S148-57

4. Söderman M, Andersson T, Karlsson B, et al. Management of patients with brain arteriovenous malformations. Eur J Radiol 2003;46:195-205

5. Hamilton MG, Spetzler RF. The prospective application of a grading system for arteriovenous malformations. Neurosurgery 1994;34: 2-7, discussion 6-7

6. Lundqvist C, Wihkolm G, Svendsen P. Embolization of cerebral arteriovenous malformations. Part II. Aspects of complications and late outcome. Neurosurgery 1996;39:460-467

7. Weber W, Kis B, Siekmann R, et al. Preoperative embolization of intracranial arteriovenous malformations with Onyx. Neurosurgery 2007;61:244-54, discussion 252-54

8. Natarajan SK, Ghodke B, Britz GW, et al. Multimodality treatment of brain arteriovenous malformations with microsurgery after embolization with Onyx: single-center experience and technical nuances. Neurosurgery 2008;62:1213-25, discussion 1225-26

9. Hurst RW, Berenstein A, Kupersmith MJ, et al. Deep central arteriovenous malformations of the brain: the role of endovascular treatment. J Neurosurg 1995;82:190-95

10. Massoud TF, Hademenos GJ. Transvenous retrograde nidus sclerotherapy under controlled hypotension (TRENSH): a newly proposed treatment for brain arteriovenous malformations-concepts and rationale. Neurosurgery 1999;45:351-63, discussion 363-65

11. Nguyen TN, Chin LS, Souza R, et al. Transvenous embolization of a ruptured cerebral arteriovenous malformation with en-passage arterial supply: initial case report. J Neurointerv Surg 2010; $2: 150-52$ 